\section{Contextualizações científicas concernentes à avaliação e diagnóstico energéticos: revisão integrativa}

\section{Sciences contextualization concerning energetic diagnostic and energy evaluation}

\section{RESUMO}

Introdução: O referido trabalho contextualiza a prática da avaliação e diagnóstico energético no processo terapêutico da Naturologia e utiliza como base de investigação os preceitos dos sistemas vitalistas de cuidado em saúde e das terapias complementares. Objetivo: Conhecer como é contextualizado a avaliação e diagnóstico energéticos nos sistemas vitalistas e nas terapias complementares. Material e métodos: Para responder ao questionamento utilizou-se do método de Revisão Bibliográfica do tipo Integrativa, na qual derivou em uma busca inicial de 130 publicações. Conforme os critérios de exclusão, apenas 03 trabalhos foram selecionados para análise, por abordarem de forma pertinente a temática da avaliações e/ou diagnósticos energéticos relacionados as terapias complementares. Resultados: Com base nos estudos analisados, foram extraídas duas categorias: (1) "Fatores de Contextualização do diagnóstico Terapêutico", que apresenta a forma como é evidenciada a dimensão diagnóstica e avaliação energética acerca das terapias complementares e (2) "Avaliação e diagnóstico energético", que discorre sobre a temática energética nos modelos diagnósticos acerca das terapias complementares apresentados. Considerações finais: Por fim, entendeu-se que o contexto das demandas socioculturais, as ferramentas utilizadas pelo terapeuta, a abordagem psicossomática, o histórico regresso do indivíduo e as percepções subjetivas, compõem a ideia de avaliação e diagnóstico energéticos utilizada pelos sistemas vitalistas, assim como pelas terapias complementares.

Palavras-chave: Anamnese. Avaliação em saúde. Diagnóstico. Terapias complementares. Vitalismo. Naturologia
CADERNOS DE

NATUROLOGIA fonusur

Adilson de Godoi

- Bacharel em Naturologia pela Universidade do Sul de Santa Catarina (UNISUL).

- Endereço: Petúnia, 362. Palhoça/SC. E-mail:a.degodoi@yahoo.com.brOrcid: https://orcid.org/0000-0002-3327-5911

Contribuições: Elaboração, concepção e delineamento do estudo, fundamentacão, coleta, análise dos dados, escrita e revisão crítica da versão final do manuscrito.

\section{Graciela Mendonça da Silva de}

Medeiros

- Enfermeira. Especialista em Acupuntura e práticas da Medicina Tradicional Chinesa. Doutora em Enfermagem pelo Programa de Pós-graduação em Enfermagem da Universidade Federal de Santa Catarina (UFSC).

- Endereço: Campus Reitor João David Ferreira Lima. Bairro Trindade.

Florianópolis/SC - CEP 88040-900 e-mail: gracielamendoncamedeiros@ gmail.com Orcid: https://orcid. org/0000-0001-5438-057X

- Contribuiçães: Elaboração, concepção e delineamento do estudo, fundamentação, supervisão das fases de execução, revisão da coleta e análise dos dados, revisão crítica da versão final do manuscrito.

Isadora Ferrante Boscoli de Oliveira Alves - Naturóloga. Mestra e Doutoranda de Enfermagem pelo Programa de Pós-graduação em Enfermagem da Universidade Federal de Santa Catarina (UFSC).

- Endereço: Campus Reitor João David Ferreira Lima. Bairro Trindade. Florianópolis/SC - CEP 88040-900 e-mail: isa.fboa@gmail.com Orcid: http://orcid.org/0000-0002-1474-6159 - Contribuições: Elaboração, concepção e delineamento do estudo, fundamentação, revisão da coleta e análise dos dados, escrita e revisão crítica da versão final do manuscrito.

\section{Carina Ceratti}

- Naturóloga. Mestra em Saúde Coletiva pelo Programa de Pós-Graduação em Saúde Coletiva da - Universidade Federal do Rio Grande do Sul (UFRGS).

- Endereco: Felizardo Furtado, 215. Porto Alegre/RS e-mail: carinaceratti@ institutoinanis.com.br Orcid: https://orcid. org/0000-0001-8771-4118

- Contribuições: Elaboração, fundamentação, escrita e revisão crítica da versão final do manuscrito.

DOI: $10.19177 /$ cntc.v8e15201927-35 


\begin{abstract}
Introduction: This research contextualizes the practice of energy evaluation and therapeutic diagnosis for science-based Naturology in the same research strand, based on the vitalist health care systems. Objective: to investigate how the energy assessment and diagnosis related to complementary therapies is contextualized. Material and method: To reach the questioning, we used the Integrative Bibliographic Review method, which resulted in an initial search of 130 publications, which according to the exclusion criteria resulted in a sample of 03 articles, because they pertinently addressed the evaluations and / or energetic diagnoses related to complementary therapies. Result: Based on the studies analyzed, two categories were presented: (1) "Contextualization Factors of Therapeutic Diagnosis", which shows how the diagnostic dimension and energetic evaluation of complementary therapies are evidenced and (2) "Energy assessment and diagnosis" that discusses the theme in the models of complementary therapies presented. Final considerations: Finally, it were conclueded that the context of socio-cultural demands, the tools used by the therapist, the psychosomatic approach, the individual's life background return and subjective perceptions wich compose up the idea of diagnosis and / or energy assessment.
\end{abstract}

Keywords: Medical History Taking. Health Evaluation. Complementary Therapies. Diagnosis. Vitalism. Naturology.

\section{INTRODUÇÃO}

A avaliação e diagnóstico são termos utilizados pelos profissionais na área da saúde para determinar o levantamento de informações, relativos aos sinais e sintomas a partir do quadro de saúde do indivíduo (avaliação) e posteriormente determinar e conhecer, a natureza do desequilíbrio, disfunção, distúrbio e até mesmo patologia (diagnóstico) para algumas profissões, como: Enfermagem, Fisioterapia, Medicina e Odontologia. O diagnóstico e avaliação energéticos procedem dos mesmos ideais de conceitos, porém utilizam como base os princípios vitalistas das Medicinas Tradicionais Chinesa, Xamânica, Ayurveda, dentre outras, assim como por outras racionalidades como a Antroposofia e a Naturologia, profissão da área da saúde no Brasil ${ }^{1-6}$.

As medicinas tradicionais vitalistas consideram a existência de uma força ou princípio vital nos seres vivos, que é responsável pela manutenção da saúde e da vida. Essa força está unida de forma indissociável ao corpo físico e sofre a influência das instâncias individuais superiores (mente, alma ou espírito). Para a concepção vitalista, a doença somente ocorre quando há um desequilíbrio desse princípio vital ou quando ocorre uma lesão física, que interfere na organização e no livre fluxo dessa energia nas partes do corpo ${ }^{7}$.

A Naturologia, por meio de sistemas terapêuticos de cuidado em saúde, como as racionalidades vi- talistas, biomédica e as práticas integrativas e complementares, propõe-se a avaliar o interagente (indivíduo que procura pelos serviços de um naturólogo) em um processo dinâmico e multidimensional ${ }^{6}$.

Conforme Teixeira (2013), os naturólogos apropriam-se de conhecimentos embasados em várias práticas de saúde vitalista, e entendem esse vitalismo como uma dimensão energética; "a percepção acerca da energia de um interagente é utilizada, pelos naturólogos, como forma de avaliação e diagnose" (TEIXEIRA, 2013, p.52) ${ }^{8}$. A avaliação do interagente faz parte das ciências clínicas, de acordo com os eixos de formação acadêmica na área de Terapias Complementares - TC, preconizado pela Organização Mundial de Saúde - OMS, bem como, suporte básico de vida, saúde pública e coletiva, além das ciências aplicadas, como bioética, relação de interagência (consulta terapêutica naturológica), racionalidades médicas vitalistas, terapias complementares, entre outras) ${ }^{9}$.

O diagnóstico naturológico apresenta uma diversidade de recursos avaliativos pautados em conceitos de bioenergia, como o uso de aparelhos de eletroneurometria (ryodoraku) e bioeletrografia. Outras práticas utilizadas como recursos avaliativos energéticos são: o mapa do corpo, técnica utilizada em arteterapia (recursos expressivos) visando empreender leituras simbólicas; teste olfativo, técnica 
utilizada em aromaterapia que considera o acesso ao inconsciente por meio do aroma mais congruente com a escolha subjetiva do interagente; o padrão reflexológico, técnica utilizada na Reflexoterapia como microssistemas podal e auricular; a Iridologia que pelo método Rayid pondera traços de personalidade, níveis de introversão e extroversão e ainda relações de ancestralidade, a forma como o sujeito se relaciona com seus pais e os padrões energéticos herdados de seus familiares; $\mathrm{e}$ as avaliações pautadas na Terapêutica Tradicional Chinesa e na Ayurveda ${ }^{10,11}$.

Por conseguinte, nota-se que há uma riqueza na prática clínica de ferramentas de avaliação e diagnóstico energéticos utilizadas e desenvolvidas no curso de Naturologia da Universidade do Sul de Santa Catarina - UNISUL, o que possibilita o planejamento e execução da terapêutica de maneira segura e eficaz. Apesar disso, identificou-se carências de publicações científicas que abordassem essa temática energética de maneira singular por parte da Naturologia. Segundo Stern (2015), “[...] o aspecto energético da terapia naturológica se mantém como uma das menos exploradas de suas publicações acadêmicas, possivelmente pela dificuldade desses profissionais em instrumentalizá-la adequadamente" ${ }^{12}$.

Perceptivelmente há uma brecha que sinaliza a necessidade de publicações científicas a respeito dos procedimentos de avaliação e diagnóstico energéticos na Naturologia, já que essa abordagem se encontra em segundo plano. Como as publicações sobre esse tema são escassas na Naturologia, optou-se por ampliar a visão e investigar sobre as avaliações e diagnósticos energéticos nas bases de dados a partir das terapias complementares $^{13}$, por abarcar os sistemas de saúde vitalistas adotados pela Naturologia em suas práticas.

Assim pretende-se com esta pesquisa responder a seguinte questão: Como é contextualizado a avaliação e diagnóstico energéticos relacionados as terapias complementares?

\section{MATERIAIS E MÉTODOS}

Trata-se de uma Revisão Bibliográfica do tipo Integrativa (RI), que consiste na construção de uma análise ampla da literatura, contribuindo para discus- sões sobre métodos e resultados de pesquisas, assim como reflexões sobre a realização de futuros estudos $^{14}$. "A revisão da literatura é um primeiro passo para a construção do conhecimento científico, pois é através desse processo que novas teorias surgem, bem como são reconhecidas lacunas e oportunidades para o surgimento de pesquisas num assunto específico". (BOTELHO; CUNHA; MACEDO, 2011) $)^{15}$.

De acordo com a metodologia, empregaram-se as seguintes etapas: 1) Identificação do tema, elaboração da hipótese ou questão de pesquisa; 2) Estabelecimento dos critérios de inclusão e exclusão e busca na literatura; 3) definição das informações a serem extraídas dos estudos selecionados; 4) avaliação dos estudos incluídos; 5) interpretação dos resultados; 6) Síntese do conhecimento ${ }^{14,15}$.

Este estudo foi conduzido a partir da questão norteadora: Como é contextualizado a avaliação e diagnóstico energéticos relacionados as terapias complementares? Foi adotado o descritor "terapias complementares" por englobar todas os sistemas de saúde que fazem referências aos sistemas de saúde vitalistas.

A busca na literatura, efetuou-se aos pares nas bases de dados: Lilacs, Pubmed, Scielo e Scopus, em agosto de 2019. Foram selecionados os estudos a partir dos descritores em ciência da saúde (DECS) ${ }^{13}$, combinados por meio de operadores (AND) e de forma trilíngue, conforme ilustrado abaixo pela Tabela 1 .

Tabela1 - Descritores utilizados conforme busca realizada nas bases de dados.

\begin{tabular}{|c|c|}
\hline Idioma & Descritores \\
\hline Português & $\begin{array}{l}\text { "Terapias complementares" AND Anamne- } \\
\text { se, “Terapias complementares" AND } \\
\text { "Avaliação em saúde”,"Terapias complemen- } \\
\text { tares"AND Diagnóstico, "Terapias comple- } \\
\text { mentares" AND Vitalismo }\end{array}$ \\
\hline Inglês & $\begin{array}{l}\text { "Complementary Therapies" AND "Medical } \\
\text { History Taking", "Complementary Thera- } \\
\text { pies" AND "Health Evaluation", "Comple- } \\
\text { mentary Therapies" AND Diagnosis, } \\
\text { "Complementary Therapies" AND Vitalism }\end{array}$ \\
\hline Espanhol & $\begin{array}{l}\text { "Terapias Complementarias" AND Anamne- } \\
\text { sis, "Terapias Complementarias" AND } \\
\text { "Evaluación en Salud”,“Terapias Comple- } \\
\text { mentarias" AND Diagnóstico,"Terapias } \\
\text { Complementarias" AND Vitalismo }\end{array}$ \\
\hline
\end{tabular}

Fonte: Elaborado por autores, Palhoça, 2019. 
Os critérios de inclusão delimitaram: documentos tipo artigos na íntegra, com resumo e texto completo disponíveis para análise, que contextualizassem a avaliação e o diagnóstico energético, nos idiomas português, inglês e espanhol, publicados no período de janeiro de 2015 a agosto de 2019. Foram excluídos artigos duplicados, que não abordaram a temática proposta, apresentados em outros idiomas à exceção do português, inglês e espanhol.

A busca resultou em 130 publicações, sendo 30 artigos na Lilacs, 19 na Pubmed, 10 na Scielo e 71 na Scopus. Após a exclusão de estudos duplicados e que não atenderam aos critérios do tema proposto, foram elegíveis 10 artigos potenciais para análise na íntegra, dos quais 02 artigos foram encontrados na base de dados Scielo, 04 na Lilacs, 02 na Pubmed e 02 na Scopus. Ao final, foram incluídos a amostra apenas 03 artigos, por abordarem a temática da avaliação e/ou diagnóstico energéticos relacionados as terapias complementares, de forma pertinente. $\mathrm{O}$ processo segue ilustrado no fluxograma da Figura 1.

Figura 1 - Fluxograma: resultados e tratamento dos artigos encontrados nas bases de dados.

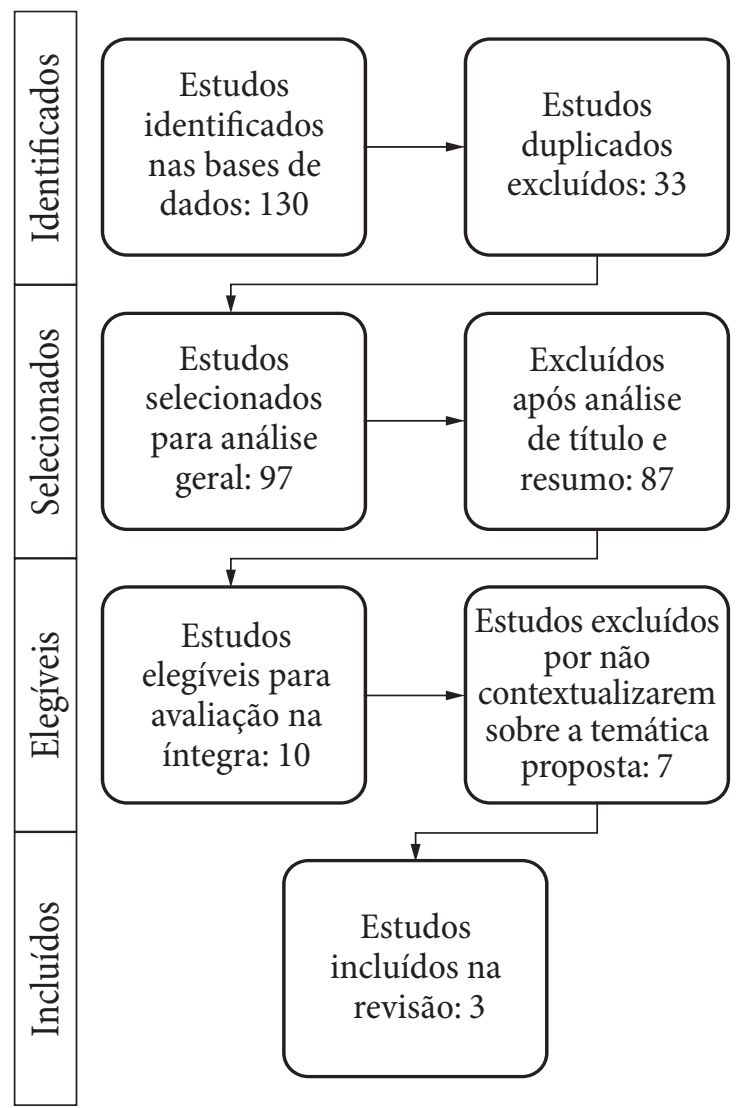

Fonte: Elaborado pelos autores, Palhoça, 2019.

\section{RESULTADO E DISCUSSÃO}

Após seleção dos artigos, foram estabelecidos critérios para extração das informações dos trabalhos, classificadas por: título do artigo; autores; título do periódico; ano de publicação e país; objetivo do estudo, delineamento e desfecho. Essas informações foram organizadas por meio do aplicativo Microsoft Office Excel, 2013, e estão ilustradas no Quadro 1.

Quadro 1 - Delineamento das informações extraídas dos estudos.

\begin{tabular}{|c|c|c|c|c|}
\hline $\begin{array}{l}\text { Artigo/ } \\
\text { Título }\end{array}$ & Autores & $\begin{array}{l}\text { Revista/ } \\
\text { Ano/ Pais }\end{array}$ & $\begin{array}{l}\text { Objetivo/ } \\
\text { Delineamento }\end{array}$ & Desfecho \\
\hline $\begin{array}{l}\text { 1. Uma } \\
\text { categoriza- } \\
\text { ção analítica } \\
\text { para estudo e } \\
\text { comparação } \\
\text { de práticas } \\
\text { clínicas em } \\
\text { distintas } \\
\text { racionalida- } \\
\text { des médicas }\end{array}$ & $\begin{array}{l}\text { Charles } \\
\text { Dalcanale } \\
\text { Tesser; } \\
\text { Madel } \\
\text { Therezinha } \\
\text { Luz }^{17}\end{array}$ & $\begin{array}{l}\text { Revista de } \\
\text { Saúde } \\
\text { Coletiva } \\
\text { (Rio de } \\
\text { Janeiro) } \\
\text { 2018, Brasil }\end{array}$ & $\begin{array}{l}\text { Propor uma } \\
\text { categorização } \\
\text { da dinâmica de } \\
\text { um encontro } \\
\text { clínico inserido } \\
\text { em ou orientado } \\
\text { por uma } \\
\text { Racionalidade } \\
\text { Médica. } \\
\text { Categorização } \\
\text { analítica }\end{array}$ & $\begin{array}{l}\text { As Racionalidades } \\
\text { Médicas fornecem } \\
\text { parâmetros para a } \\
\text { avaliação das } \\
\text { ações terapêuticas } \\
\text { na sua própria } \\
\text { perspectiva, assim } \\
\text { como dos terapeu- } \\
\text { tas para a } \\
\text { interpretações } \\
\text { (diagnoses) e } \\
\text { reavaliação dos } \\
\text { doentes. }\end{array}$ \\
\hline $\begin{array}{l}\text { 2. Medicina } \\
\text { Tradicional } \\
\text { Comple- } \\
\text { mentar e } \\
\text { Integrativa: } \\
\text { desafios } \\
\text { para construir } \\
\text { um modelo } \\
\text { de avaliação } \\
\text { do cuidado }\end{array}$ & $\begin{array}{l}\text { Islândia } \\
\text { Maria } \\
\text { Carvalho } \\
\text { de Sousa; } \\
\text { Virginia } \\
\text { Alonso } \\
\text { Hortale; } \\
\text { Regina } \\
\text { Cele de } \\
\text { Andrade } \\
\text { Bodstein18 }\end{array}$ & $\begin{array}{l}\text { Tema livre } \\
\text { 2016, Brasil }\end{array}$ & $\begin{array}{l}\text { Propor } \\
\text { elementos para a } \\
\text { construção de } \\
\text { um modelo de } \\
\text { avaliação que } \\
\text { contribua com a } \\
\text { pluralidade da } \\
\text { Medicinas } \\
\text { Tradicionais } \\
\text { Complementares } \\
\text { e Integrativas } \\
\text { nas diversas } \\
\text { dimensões do } \\
\text { cuidado em } \\
\text { saúde. Revisão } \\
\text { narrativa da } \\
\text { literatura }\end{array}$ & $\begin{array}{l}\text { É necessário } \\
\text { a construção } \\
\text { de modelos } \\
\text { avaliativos que } \\
\text { não ignorem } \\
\text { as diversas } \\
\text { racionalidades } \\
\text { que atravessam o } \\
\text { processo } \\
\text { saúde- doença. } \\
\text { Modelos mais } \\
\text { apropriados e } \\
\text { factíveis, } \\
\text { precisariam ser } \\
\text { discutidos, o que } \\
\text { pressupõe a } \\
\text { ampliação do } \\
\text { debate que ainda é } \\
\text { incipiente no país. }\end{array}$ \\
\hline $\begin{array}{l}3 . \\
\text { O movimento } \\
\text { Yīn e Yáng na } \\
\text { cosmologia } \\
\text { da medicina } \\
\text { chinesa }\end{array}$ & $\begin{array}{l}\text { Bernardo } \\
\text { Diniz } \\
\text { Coutinho; } \\
\text { Pérola } \\
\text { Goretti } \\
\text { Sichero } \\
\text { Dulcetti19 }\end{array}$ & $\begin{array}{l}\text { História, } \\
\text { Ciências, } \\
\text { Saúde } \\
\text { Manguinhos } \\
\text { 2015, Brasil }\end{array}$ & $\begin{array}{l}\text { Aprofundar o } \\
\text { conhecimento } \\
\text { sobre o aspecto } \\
\text { do movimento } \\
\text { Yinn e Yáng por } \\
\text { intermédio da } \\
\text { perspectiva da } \\
\text { cosmologia } \\
\text { daoista e da } \\
\text { medicina } \\
\text { tradicional } \\
\text { chinesa, } \\
\text { buscando } \\
\text { conhecer sua } \\
\text { teoria e } \\
\text { contribuições } \\
\text { para o sistema } \\
\text { diagnóstico e } \\
\text { terapêutico da } \\
\text { medicina } \\
\text { tradicional } \\
\text { chinesa e da } \\
\text { acupuntura. } \\
\text { Revisão } \\
\text { bibliográfica da } \\
\text { literatura }\end{array}$ & $\begin{array}{l}\text { No modelo } \\
\text { tradicional chinês, } \\
\text { entende-se a } \\
\text { patologia como } \\
\text { uma desarmonia } \\
\text { no movimento } \\
\text { Yĩn e Yáng na vida } \\
\text { de um indivíduo, } \\
\text { partindo da noção } \\
\text { de que o mesmo } \\
\text { está em } \\
\text { ressonância com } \\
\text { o universo. } \\
\text { O diagnóstico } \\
\text { consiste na } \\
\text { percepção dos } \\
\text { sinais dessa } \\
\text { perturbação e o } \\
\text { cuidado se dá por } \\
\text { meio do incentivo } \\
\text { ao autoconheci- } \\
\text { mento, além da } \\
\text { promoção de } \\
\text { hábitos de vida } \\
\text { saudáveis. }\end{array}$ \\
\hline
\end{tabular}

Fonte: Elaborado pelos autores, Palhoça, 2019. 
Após análise e interpretação dos dados, os artigos foram categorizados para melhor elucidar o entendimento e as atribuições deduzidas, sendo a primeira categoria: "Fatores de contextualização do diagnóstico terapêutico", que apresentam fatores norteadores do diagnóstico e avaliação relacionados as terapias complementares. A outra categoria: "Avaliação e diagnóstico energético", discursa sobre a abordagem vitalista, energética, das avaliações e diagnóstico praticados acerca das terapias complementares.

\section{FATORES DE CONTEXTUALIZAÇÃO DO DIAGNÓSTICO TERAPÊUTICO}

Primeiramente, notou-se que os três estudos apresentam um modelo ideal de conduta terapêutica, praticado cada um de acordo com os sistemas de cuidado em saúde que demonstram utilizar. $\mathrm{O}$ estudo 01 (referindo-se ao quadro de resultados descrito acima) apresenta, com base nas racionalidades médicas (todo o sistema médico complexo construído sobre seis dimensões: uma morfologia humana, uma dinâmica vital, uma doutrina médica, um sistema diagnóstico, uma cosmologia e um sistema terapêutico) seu modelo ideal de ato clínico, construído a partir da eficácia simbólica, em que o fluxo da ação clínica pode ser decomposto, para fins de análise em seis movimentos: acolhida e escuta (a pessoa que se sente doente como protagonista); investigação e elaboração da interpretação (aqui o protagonismo fica a cargo do terapeuta/ curador); socialização do diagnóstico; elaboração e execução do tratamento (nestas etapas são consideradas as experiências de ambos) $)^{16,17}$.

O estudo 2, apresenta as seguintes características: singularidade (o foco do cuidado é a pessoa e não a doença); flexibilidade (adequação do cuidado ao usuário e seu contexto); complexidade (o processo de adoecimento engloba o desequilíbrio na dinâmica vital, no ser, além do físico, químico e biológico) e integralidade (a intervenção e sua avaliação contemplam resultados objetivos e subjetivos, não basta curar os sintomas da enfermidade $)^{18}$.

O estudo 03 disserta sobre a doutrina médica chinesa, especificamente, os movimentos yin e yang, que compõe um dos fundamentos básicos da sabe- doria chinesa antiga. Esse fundamento é utilizado para descrever o estado dinâmico de equilíbrio do sopro vital (energia vital) dentro e fora do corpo da pessoa, seja no funcionamento correto de seus órgãos e funções orgânicas, bem como na sua relação com o meio ambiente). Apesar do estudo não apresentar em etapas o processo diagnóstico, como nos estudos 02 e 03 , ele o descreve por meio dos conceitos yin e yang, que conferem uma dinâmica cíclica da qual se caracteriza a dimensão energética, relacionando os movimentos que harmonizam os desequilíbrios apresentados quando há enfermidade ${ }^{19}$.

Outro fator que pode ser evidenciado nos três estudos como convergente é uma dinâmica orbital no processo avaliativo e diagnóstico, caracterizada a partir dos primeiros passos dados em direção ao terapeuta, seguidos de uma relação entre ambos de forma o mais horizontal possível e priorizando uma comunicação ideal correlacionando o ponto de vista de cada um. Ao final, respeita-se a decisão do tratamento que foi selecionado a partir da experiência profissional do terapeuta, evitando desconforto, mas levando em conta as experiências do indivíduo. ${ }^{17,18,19}$ Esse processo por ser dinâmico, sofre a todo momento alterações de acordo com novas manifestações, o estudo 02 sinaliza que alguns efeitos são avaliados durante o processo e, tanto o diagnóstico quanto os objetivos terapêuticos, podem ser reformulados e reavaliados, como ocorre em algumas racionalidades médicas ${ }^{18}$.

Porém, uma racionalidade médica que, de acordo com os 03 artigos analisados, intercepta essa dinâmica no processo diagnóstico é a biomedicina. Conforme o estudo 02, as dimensões do cuidado na Medicina Tradicional Complementar e Integrativa exigem que seus resultados sejam verificados tanto a partir da manifestação física, quanto a partir da maneira como o usuário se sente. Diferente, portanto, da biomedicina em que os resultados são predominantemente verificados a partir da cessação de sintomas da doença. O paradigma biomédico, desenvolveu seu pensamento no conceito dualista de normal ou patológico, que se preocupa mais com a doença e o diagnóstico do que com o doente e o cuidado ${ }^{17,18,19}$. 
Neste contexto, diversos fatores, poderiam responsabilizar as condutas mecanicistas da biomedicina, que prioriza a agilidade e eficácia de ferramentas de avaliação e diagnóstico, assistindo o paciente em segundo plano. Tais fatores, também contribuíram para o aumento da oferta e uso dos sistemas de cuidado com terapias complementares. Estas mudanças são responsáveis tanto pelo aparecimento de síndromes e transtornos relacionados à ansiedade, insônia, dores crônicas, estresse e depressão, déficit de atenção, entre outros, quanto pela maior longevidade da população, que responde em grande parte pela prevalência das doenças crônico-degenerativas ${ }^{17,18}$.

Dessa forma, as terapias complementares ofertam uma diversidade no cuidado em saúde, desagravando uma demanda que não pode ser assistida em sua integridade, como ocorre no processo dinâmico terapêutico. Foi observado que há uma parte mais importante e que fica a cargo da racionalidade atuante no momento e/ou do protagonismo do terapeuta. Dada a pluralidade das racionalidades médicas, um mesmo 'caso', potencialmente admitirá mais de uma interpretação. Os saberes das várias racionalidades médicas, exercitados pelos seus curadores, entram em jogo de modo a produzir a diversidade de interpretações (e tratamentos) das racionalidades médicas que persistem no mundo ${ }^{18}$.

Consequentemente, a visão que se tem de diagnóstico acerca das terapias complementares, varia de acordo com sistema de cuidado a que se refere, seu contexto sociocultural e particularidades que dependem exclusivamente do terapeuta, considerando sua experiência, além da relação com o sujeito cuidado. Desse modo, outro fator apontado aqui é a aplicabilidade do processo de diagnóstico terapêutico, que pôde ser melhor vislumbrado a partir do estudo 03 , talvez por se tratar especificamente da racionalidade médica Chinesa. Uma vez que o adoecimento é processo em contínua modificação, o diagnóstico visa identificar o padrão de desequilíbrio do Qi (definido aqui somente como sopro vital, já que esta definição está desprovida do conceito filosófico tradicional chinês) no intuito de antecipar a evolução da enfermidade, utilizando-se de oito princípios tradicionais. O excesso ou a insuficiência permanente do Qi são as causas fundamentais para o aparecimento e o desenvolvimento de enfermidades, sendo necessário discernir o Yìn do Yáng durante a elaboração do diagnóstico ${ }^{18,19}$.

\section{AVALIAÇÃO E DIAGNÓSTICO ENERGÉTICO}

O artigo 02, traz a definição de que a leitura (avaliação) ou o diagnóstico do processo de adoecimento leva em conta o fluxo de energia, emoções, alimentação, atividades, relacionamentos, expressões, sintomas e características físicas, contexto cultural e social. Considera ainda que cada indivíduo tem uma constituição e circunstâncias psicossociais próprias, reações distintas diante dos sintomas, doenças e tratamentos. Portanto, o adoecer aqui, não é consequência somente de desequilíbrios biológicos e bioquímicos, mas a maneira como o indivíduo se posiciona e interage com seu contexto, como se relaciona consigo mesmo e com o adoecimento ${ }^{18}$.

Essa definição pode ser observada na prática em um exemplo citado no estudo 03: Na China antiga, havia um revezamento de trabalho entre as tecelãs, que passavam o inverno preparando tecidos para a estação da primavera, enquanto os homens repousavam em cavernas para restabelecer as suas "energias". Já na primavera, os homens partiam para passar o verão trabalhando no campo "ativando-se ao sol", e as tecelãs abrigavam-se. Observa-se aqui que as palavras "energias", denotam vigor físico, disposição e até saúde. E que "ativando-se ao sol” se estimula a vitalidade e força. Porém esse conceito de energia não existia para o chinês antigo, mas é o mais próximo que se possa encaixar na visão ocidental de energia atualmente ${ }^{19}$.

Essa energia nem sempre está impressa visivelmente nas palavras, mas sim nas atribuições a ela, como elementos que são usualmente utilizados como informações no levantamento de dados (avaliação) ou na tomada de decisão, conferindo um diagnóstico energético em saúde. As dimensões do cuidado na Medicina Tradicional Complementar e Integrativa, apresentados pelo estudo 02, exigem 
que seus resultados sejam verificados tanto a partir da manifestação física, quanto a partir da maneira "como o usuário se sente", diferente, portanto, da biomedicina em que os resultados são predominantemente verificados a partir da cessação de sintomas da doença. Neste sentido, a doença é entendida como um processo natural de evolução que pode ser vista como um desequilíbrio do QI na racionalidade chinesa, da dinâmica vital na racionalidade homeopática, desequilíbrio do Prana (energia vital, na racionalidade Ayurveda) ou da polarização de energia na terapia floral ${ }^{18}$.

Interessante notar que o estudo 03, traz uma comparação energética, inter-relacionando yin/yang e terapeuta/paciente no processo de cura, em que o sujeito cuidado passivo à intervenção é a face Yinn, e o terapeuta que a executa é a contraparte Yáng, que manifesta em seu ato a intenção de reestabelecer a harmonia da circulação do $Q i$ no sujeito cuidado, devendo respeitar a ordem de inserção e retirada das agulhas na acupuntura (a acupuntura tem por finalidade harmonizar o fluxo do Qi por meio da estimulação, através de agulhas entre outros, de pontos apropriados no corpo) utilizadas para amenizar os sintomas decorrentes do desequilíbrio ${ }^{19}$.

A partir das terapias complementares, cada racionalidade médica fornece parâmetros para avaliação das ações terapêuticas na sua própria perspectiva, que são, via de regra, os mesmos usados pelos seus terapeutas para a produção das interpretações (avaliação e diagnóstico) e reavaliação dos estados de saúde. Assim, o ser doente, via de regra, aceita a interpretação, acreditando no saber especializado do curador, dado haver nela elementos convergentes com suas crenças e modelos explicativos. Essa socialização da interpretação envolve e engaja o ser doente, induz nele uma reorganização simbólica e emocional (acalma medos, angústias e ansiedades) e reorganizando seus sentidos, preparando o tratamento, coerentemente com a interpretação. Tal interpretação ressignificará o quadro trazido pelo ser doente, dando-lhe sentido e significado atrelados aos saberes, valores e técnicas do curador e a racionalidade médica que utiliza ${ }^{17,18}$.
Por vezes, dentro de uma mesma racionalidade várias interpretações podem ser produzidas com efetividade, nos tratamentos delas decorrentes, conforme as situações e os terapeutas. Na medida em que a intervenção não se circunscreve à doença, a cura ou o cessar dos sintomas não é o único objetivo a ser alcançado. Avaliar e diagnosticar a partir das terapias complementares, portanto, não se resume em identificação ou interpretação da enfermidade, visto que se leva em conta a integridade do indivíduo. $O$ processo de cura pode ser definido como uma trajetória de autoconhecimento e crescimento e os resultados são analisados levando em conta outras evidências (energias), como a ampliação da sensação de bem-estar e felicidade ${ }^{17,18}$.

\section{CONSIDERAÇÕES FINAIS}

A pesquisa foi estabelecida a partir da premissa de que a Naturologia, mesmo utilizando de recursos diversificados em sistemas vitalistas de cuidado em saúde, apresenta escassez de trabalhos relacionados a avaliação e diagnóstico energéticos, em suas produções científicas. Assim sendo, ampliou-se o olhar e a busca para as terapias complementares, por englobar os sistemas de cuidado em saúde utilizados, também, pela Naturologia no Brasil.

No entanto, salienta-se que esta temática energética, possivelmente por sua subjetividade, é pouco explorada de maneira singular no meio científico em produções a partir da área da saúde no Brasil. Observa-se nesse estudo a necessidade das profissões da área da saúde, que utilizam de sistemas de cura vitalistas se apropriarem de modelos de avaliação e diagnóstico energéticos e se inclinem numa linguagem que possa uniformizar as subjetividades relacionadas as manifestações da energia vital no indivíduo. Permitirem-se a apropriação, para além da prática clínica, dos sistemas de avaliação e diagnose energética aqui abordados aprofundando-se, também, no campo científico. Para poder dialogar e integrar o sistema de saúde vigente. Sendo esse artigo pioneiro nessa abordagem voltada para a Naturologia, profissão no Brasil que utiliza das terapias complementares. 
Por meio dos estudos explorados, pôde-se compreender de que forma são contextualizados a avaliação e o diagnóstico energético acerca das terapias complementares, que abrangem diferentes sistemas de cura vitalista. Observou-se que a avaliação energética está inserida no processo dinâmico do diagnóstico terapêutico e que o cunho energia nem sempre está impresso nas ferramentas de avaliação, podendo ser identificada por diversos fatores subjetivos como: o olhar do terapeuta, a simbologia da patologia, as demandas socioculturais, emoções, os relacionamentos, as características físicas, bem como as relações psicossomáticas e de personalidade de cada pessoa.

Posteriormente evidenciou-se que o diagnóstico terapêutico energético é dinâmico e orbita baseado nessa gama de informações que levam em conta ainda a experiência profissional do terapeuta, as práticas que utiliza, seu olhar clínico e a relação terapeuta-interagente. $\mathrm{O}$ que garante essa abordagem é, portanto, a relação terapeuta-interagente construída de modo a agrupar características singulares dos sujeitos, tendo em conta suas relações socioculturais in-

\section{CONFLITOS DE INTERESSE}

Declaram não haver.

\section{FONTES DE FINANCIAMENTO}

Declaram não haver.

\section{REFERÊNCIAS}

1. Peres HHC, Jensen R, Martins TYC. Avaliação da acurácia diagnóstica em enfermagem: papel versus sistema de apoio à decisão. Acta Paul. Enf. 2016 Abr; 29(2): 218-224.DOI: <http://dx. doi.org/10.1590/1982-0194201600030>.

2. Braz RG; Goes F, Pedroso DC, Carvalho GA. Confiabilidade e validade de medidas angulares por meio do software para avaliação postural. Fisioterapia em Movimento, 2017 set; 21(3). ISSN 1980-5918. Disponível em: <https://periodicos.pucpr.br/index.php/ fisio/article/view/19185/18509>. Acesso em: 23 out. 2019.

3. Sancho KA, Pfeiffer CRC, Correa CRS. Medicalização, diagnóstico clínico e queixa-conduta - redes de significação em jogo. Interface (Botucatu), Botucatu. 2019 out; 23(170633). DOI: <http://dx.doi. org/10.1590/interface.170633

4. Rodrigues LP et al. Uso de métodos não invasivos para avaliação da espessura muco-gengival: a tecnologia a favor do diagnóstico. $\mathrm{Hu}$ Revista. 2019 fev; 44(1): 23-28. http://dx.doi.org/10.34019/19828047.2018.v44.13952.

5. Follador E. Medicina antroposófica: um novo paradigma para as questões da medicina moderna. Rev. Med. (São Paulo) [Internet]. 30set.2013 [citado 12set.2019];92(3):166-72. Available from: http:// www.revistas.usp.br/revistadc/article/view/79997. dependentes. Retrata-se que tais características conferem um diagnóstico dinâmico (energético), usualmente utilizado também pela Naturologia no Brasil.

Um dos fatores, observado nos artigos e que, demonstra limitação, é a distinção entre o entendimento e a abordagem do contexto saúde-doença, o que acarreta na dificuldade de integração do modelo vigente de cuidado em saúde, por não reconhecer e/ ou validar outros aspectos de diagnóstico e avaliação. Como, por exemplo, a psicossomática e longevidade, que por serem subjetivas e únicas para cada indivíduo podem contribuir, tanto para a emergência (sendo consideradas e auxiliarem na abordagem do modelo biomédico), quanto para a negligência (no sentido de que quando não considera poder levar a tratamentos e intervenções que seriam desnecessárias).

Por fim, entende-se que a contextualização da avaliação e diagnóstico energéticos voltados para as terapias complementares, merece espaço no campo da saúde, das práticas de cunho vitalistas e na condução terapeuta-paciente/interagente, sugerindo-se, portanto, estudos que elaborem essas dimensões.

6. Sabbag SHF et al. A Naturologia no Brasil: avanços e desafios. Cad. Naturol. Terap. Complem. 2(2):11-32, jan./jun. 2013.

7. Teixeira M. Antropologia Médica Vitalista: uma ampliação ao entendimento do processo de adoecimento humano. Rev. Med.29set.2017 [citado 12nov.2019];96(3):145-58. Available from: http://www.revistas.usp.br/revistadc/article/view/110789.

8. Teixeira, Diogo Virgílio. Integralidade, interagência e educação em saúde: uma etnografia da naturologia. (Dissertação de mestrado em Antropologia). Programa de Pós Graduação em Antropologia Social, Universidade Federal de Santa Catarina, Florianópolis, 2013.

9. Rodrigues DMO, Wedekin LM. Graduação em naturologia: um diferencial na formação de um profissional do cuidado. Cad. Naturol. Terap. Complem; v. 2, n.2, p. 09-10, jan./jun. 2013

10. Ceratti C, Hellmann F. Proximidades e distanciamentos entre as formações de naturologia e naturopatia à luz do conceito de racionalidades médicas. 2015.26 f. TCC (Graduação) - Curso de Naturologia, Universidade do Sul de Santa Catarina, Palhoça, 2015.

11. Guerriero, S; STERN, Fábio L. The new age Concepts of Energy: the case of the Brazillian Naturology. Caminhos. 2017 out; 15(1): 4-25. Pontificia Universidade Catolica de Goias -PUC Goias. http://dx. doi.org/10.18224/cam.v15il.5963 
12. Stern, Fábio L. Naturologia e espiritualidade: indícios dos valores do movimento da Nova Era entre naturólogos formados no Brasil. 2015. 224 f. (Dissertação Programa de Pós-Graduação em Ciências da Religião) - Pontifícia Universidade Católica de São Paulo, São Paulo, 2015.

13. Descritores em Ciências da Saúde: DeCS. 2019. ed. rev. e ampl. São Paulo: BIREME / OPAS / OMS, 2017. Disponível em: < http://decs. bvsalud.org >. Acesso em 22 de ago. 2019.

14. Mendes, K. D. S., Silveira, R. C. D. C. P., \& Galvão, C. M. (2008). Integrative literature review: a research method to incorporate evidence in health care and nursing. Texto \& ContextoEnfermagem, 17(4), 758-764.

15. Botelho LLR, Cunha CC de A, Macedo M. O método da revisão integrativa nos estudos organizacionais. GeS [Internet]. $2^{\circ} \mathrm{de}$ dezembro de 2011 [citado $12^{\circ}$ de novembro de 2019];5(11):121-36. Disponível em: https://www.gestaoesociedade.org/ gestaoesociedade/article/view/1220
16. Luz, Madel Therezinha; BARROS, Nelson Felice. Racionalidades Médicas e Práticas Integrativas em Saúde: estudos teóricos e empíricos. Rio de Janeiro: UERJ/IMS/LAPPIS, 2012.

17. Tesser CD, Luz MT. Uma categorização analítica para estudo e comparação de práticas clínicas em distintas racionalidades médicas. Physis [Internet]. 2018 Mar [citado 2019 Out 24] ; 28(1 ): e280109. http://dx.doi.org/10.1590/s0103-73312018280109

18. Sousa IMC et al. Medicina Tradicional Complementar e Integrativa: desafios para construir um modelo de avaliação do cuidado. Ciência \& Saúde Coletiva; 2018 out 23(10): 3403-3412. FapUNIFESP (SciELO). http://dx.doi.org/10.1590/1413812320182310.23792016

19. Coutinho BDD, Pérola GS. O movimento Yīn e Yáng na cosmologia da medicina chinesa. Hist. cienc. saude-Manguinhos; 2015 set. 22(3): 797-811 Rio de Janeiro. http://dx.doi.org/10.1590/ S0104-59702015000300008 